\title{
Experiência do Usuário (UX): Análise Bibliométrica de publicações sobre a Avaliação de Desempenho da UX
}

\author{
User Experience: Bibliometric Analysis of Publications on UX Performance Evaluation
}

DEMILIS, Marcelo P.; Mestre; Universidade Federal de Santa Catarina

marcelodemilis@gmail.com

MATOS, Lucas S.; Mestre; Universidade Federal de Santa Catarina

lukxmatos@gmail.com

ENSSLIN, Sandra R.; Doutora; Universidade Federal de Santa Catarina

sensslin@gmail.com

MERINO, Giselle S. A. D.; Doutora; Universidade Federal de Santa Catarina; Universidade da Região de Joinville

gisellemerino@gmail.com

\section{Resumo}

A busca por métodos para mensurar a Experiência do Usuário (UX) aumentou progressivamente junto com um crescente interesse no design orientado para a experiência. Contudo, as experiências abrangem uma gama de propriedades e métricas que são extremamente variadas, sendo particulares, muitas vezes efêmeras e subjetivas (HASSENZAHL, M.; SCHÖBEL, M.; TRAUTMANN, T, 2008; HASSENZAHL, M; DIEFENBACH, S; GÖRITZ, 2010), dificultando sua categorização, definição e mensuração. Adotando-se o instrumento de intervenção ProKnow- $C$, objetivou-se investigar as pesquisas científicas, em língua inglesa, sobre Avaliação de Desempenho da Experiência do Usuário, a fim de identificar lacunas e oportunidades de pesquisa. Selecionaram-se 77 artigos, entre 2000 e 2017, os quais permitiram identificar o panorama geral das publicações relevantes, periódicos e autores prolíficos para a temática.

Palavras-chave: Avaliação de Desempenho, Experiência do Cliente, Experiência do Usuário, Indicador, Perspectiva do Usuário, Revisão de Literatura.

\section{Abstract}

The search for methods to measure User Experience (UX) has progressively increased along with a growing interest in experience-oriented design. However, the experiments cover a range of properties and metrics that are extremely varied, being particular, often ephemeral and subjective (HASSENZAHL, M.; SCHÖBEL, M.; TRAUTMANN, T, 2008; HASSENZAHL, M; DIEFENBACH, S; GÖRITZ, 2010), making it difficult to define and measure them. Therefore, by adopting the ProKnow-C intervention instrument, the objective was to investigate the English-language scientific research on Performance Evaluation of the User Experience and the research gaps and opportunities based on the results of bibliometric and systemic analysis. 77 articles were selected between 2000 and 2017, analyzing and exposing the general panorama of relevant publications, periodical and prolific authors for the theme.

Keywords: Performance Evaluation, Customer Experience, User Experience, Indicator, User Perspective, Literature Review. 


\section{Introdução}

A busca por métodos para mensurar a Experiência do Usuário (UX) aumentou progressivamente junto com o crescente interesse no design orientado para a experiência. Em um primeiro momento, designers pesquisadores adotaram instrumentos emprestados das Ciências Sociais para realizar essas medidas, sendo posteriormente desenvolvidos instrumentos dedicados a esses domínios (LAURANS et al., 2009; POELS; DEWITTE, 2006; TONETTO, L. M.; DESMET, P. M. A, 2016). Contudo, as experiências abrangem uma gama de propriedades e métricas, sendo particulares, muitas vezes efêmeras e subjetivas (LAW et al. Al, 2009; HASSENZAHL, M; DIEFENBACH, S; GÖRITZ, 2010), portanto condicionadas aos fatores humanos (GREEN; JORDAN, 1999), tornando difícil sua definição e mensuração mais precisa. Por isso a dificuldade de encontrar conceitos concisos que delimitem os aspectos que compõem a experiência do usuário e, consequentemente, ferramentas que explorem esses aspectos.

Sendo a experiência do usuário projetável (HASSENZAHL, M; DIEFENBACH, S; GÖRITZ, 2010), para construí-la de forma que seja positivamente significativa e memorável, é preciso considerar todos os aspectos que a compõem e como eles se formam. A Avaliação de Desempenho (AD), nesse contexto, pode trazer muitos benefícios, de forma que os indicadores construídos e organizados como Sistemas de AD forneçam feedback tempestivo aos gestores das organizações (LEBAS, 1995; NEELY; GREGORY; PLATTS, 1995), estimulando a melhoria contínua da UX. Porém, as respostas dadas pelos Sistemas de AD somente são efetivas se as métricas individuais estiverem alinhadas aos objetivos e às estratégias da organização (GHALAYINI; NOBLE, 1996; FERREIRA; OTLEY, 2009).

Por isso, é fundamental que haja entendimento dos ambientes interno e externo em que a organização está inserida, suas estratégias (MELNYK et al., 2014; CARNEIRO-DA-CUNHA; HOURNEAUX; CORRÊA, 2016), estando estas alinhadas com as percepções, expectativas e necessidades do usuário. A AD poderá possibilitar, sob uma perspectiva construtivista, o acompanhamento dos indicadores da UX, trazendo inúmeras vantagens nesse contexto.

Nesse sentido, emerge a seguinte questão-problema: Quais as lacunas e oportunidades de pesquisa identificadas na literatura sobre AD da Experiência do Usuário? Desse modo, objetiva-se, no presente artigo, investigar as pesquisas científicas, em língua inglesa, sobre Avaliação de Desempenho da Experiência do Usuário, a fim de identificar lacunas e oportunidades de pesquisa. Para a orientação da seleção, reflexão e crítica do Portofólio Bibliográfico (PB), adotou-se o Knowledge Development Process-Constructivist (ProKnow-C) (ENSSLIN; ENSSLIN; PINTO, 2013; VALMORBIDA; ENSSLIN, 2017; THIEL; ENSSLIN; ENSSLIN, 2017) como instrumento de intervenção.

Este estudo justifica-se quanto à sua importância e viabilidade (CASTRO, 1977), fornecendo um panorama geral do fragmento da literatura e a identificação de possíveis avanços nessa área de conhecimento com base na análise dos artigos do PB. É original pela carência de estudos que permitem a geração de conhecimento com análise das lacunas existentes e oportunidades de pesquisas futuras, pois estudos empíricos evidenciam que a $A D$ ocasiona melhoria no desempenho e produtividade na organização (BROWN; HYATT; BENSON, 2010), e a Experiência do Usuário resulta em benefícios para a empresa pelo produto ou serviço oferecido (UNGER; CHANDLER, 2009). Quanto à viabilidade, houve o interesse dos pesquisadores na busca de conhecimento nessa área e pela acessibilidade de artigos disponíveis no Portal de Periódicos da CAPES. Em relação à delimitação de pesquisa, utilizaram-se somente artigos científicos em língua inglesa, no período de 2000 a 2017. 


\section{Metodologia: Enquadramento metodológico e procedimentos de coleta e tratamento dos dados}

A pesquisa desenvolvida possui caráter qualitativo (CRESWELL, 2014) por apresentar como objetivo a geração de conhecimento e a análise crítica a respeito da Avaliação de Desempenho da Experiência do Usuário e evidenciar oportunidades de pesquisa para a comunidade científica. No que se refere aos procedimentos técnicos, trata-se de uma pesquisa-ação e bibliográfica (CRESWELL, 2014) por ser um processo que envolve aprendizado e modificação até se obtiverem dados pertinentes à análise desejada com base nos artigos científicos selecionados. A validade dos procedimentos e confiabilidade dos resultados foram feitos por meio de member checking que conferiu todo o processo desenvolvido e os dados coletados (CRESWELL, 2014), e também código de verificação cruzada, realizada por dois dos autores da presente pesquisa. A pesquisa foi feita com base na seleção e análise de artigos publicados em língua inglesa no Portal de Periódicos da CAPES.

Foi feita a coleta de dados primários e secundários. Os dados primários foram obtidos diretamente das delimitações dos pesquisadores desde a busca nas bases de dados para seleção dos artigos que irão compor o Portfólio Bibliográfico (PB) como nas variáveis a serem analisadas (RICHARDSON, 1999). Por sua vez, os dados secundários são os artigos do PB, de onde as variáveis foram identificadas e analisadas (DUTRA et al., 2015). O instrumento de intervenção aplicado é o processo estruturado para seleção, reflexão e análise de um conjunto de artigos selecionado na literatura, denominado Knowledge Development Process-Constructivist (ProKnow-C). Dutra et al. $(2015$, p. 7) afirmam que o "o ProKnow-C é usado com o intuito de construir conhecimento para determinado pesquisador com base em seus interesses, escolhas e delimitações, segundo uma visão construtivista". O ProKnow-C é composto por quatro etapas: (1) seleção do PB; (2) bibliometria; (3) análise sistêmica; e (4) formulação da pergunta e objetivos da pesquisa (DUTRA et al., 2015; LACERDA et al., 2014). Em razão de o pesquisador interagir diretamente em todas as etapas da pesquisa, o caráter subjetivo é percebido na composição desse fragmento de literatura (VALMORBIDA; ENSSLIN, 2017; ENSSLIN et al. 2014; LACERDA; ENSSLIN; ENSSLIN, 2012).

Para alcançar o objetivo desta pesquisa, selecionou-se o PB com base na combinação de palavras-chave de dois eixos: Avaliação de Desempenho e Experiência do Usuário (Figura 1).

Figura 1. Resultados das buscas das bases de dados.

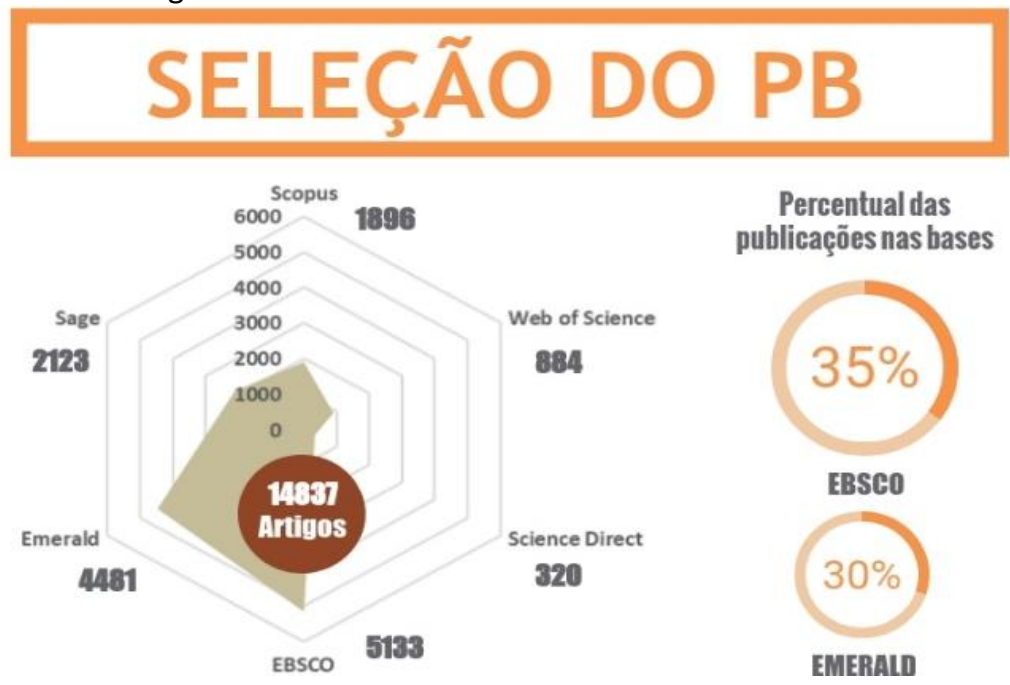

Fonte: Elaborado pelos autores. 
As buscas foram feitas entre 29 de setembro e 15 de outubro de 2017 . O resultado das buscas nas bases de dados é apresentado na Figura 1, e a operacionalização da etapa de Seleção do Portfólio Bibliográfico e os resultados são apresentados na Figura 2.

Figura 2. Resultados das buscas das bases de dados.

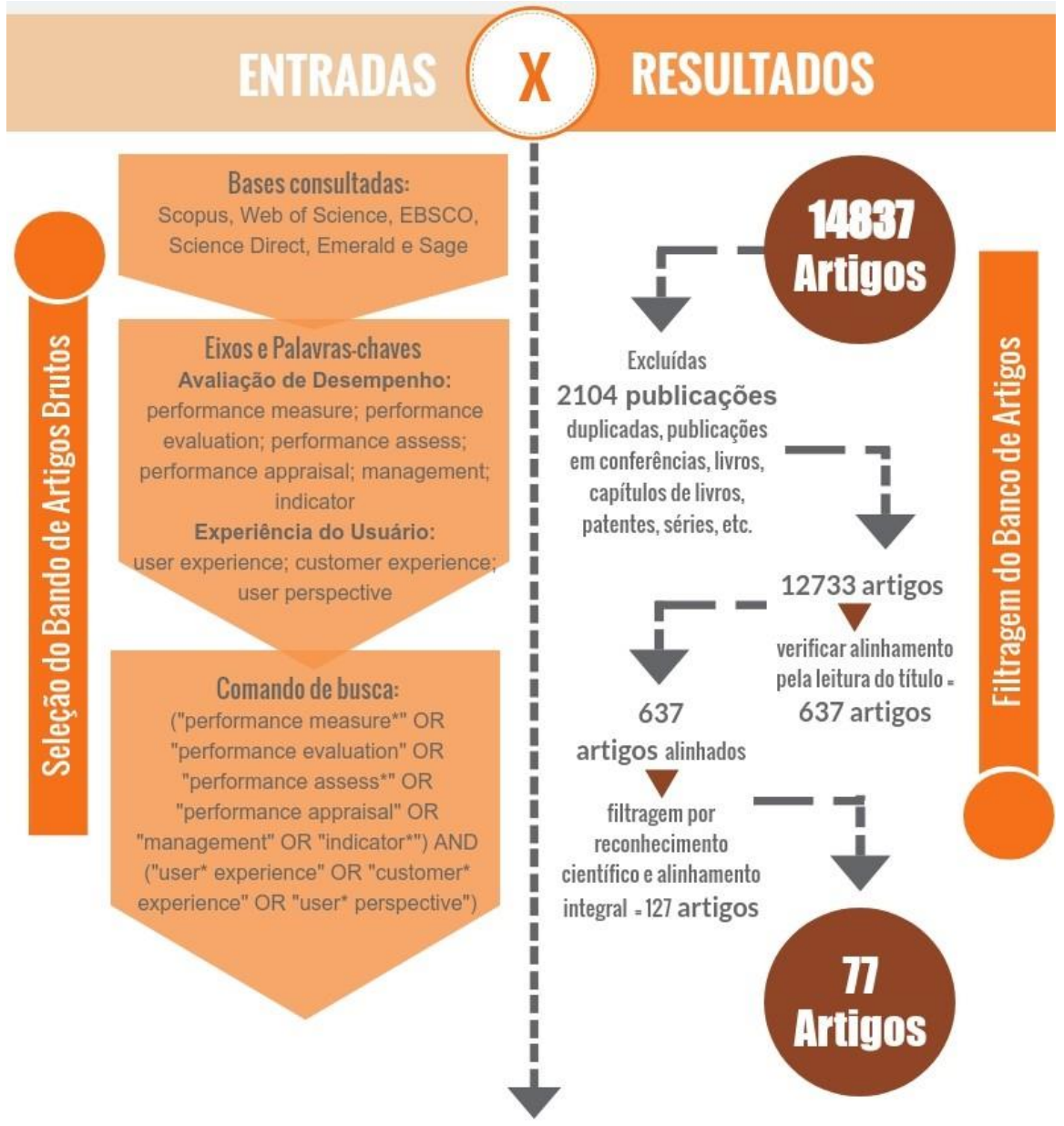

Fonte: Elaborado pelos autores.

Com base no PB selecionado, fez-se a segunda etapa do ProKnow-C: análise bibliométrica, que objetivou identificar e evidenciar o destaque de certas características (variáveis delimitadas) do PB analisado e de suas referências. Para tratamento das variáveis, foi feita a contagem de ocorrências e/ou elementos que permitiram ampliar o conhecimento sobre o tema. Na sequência, procedeu-se à interpretação dos dados obtidos para garantir a geração de conhecimento; buscouse sua origem e dados complementares que justificassem as ocorrências (THIEL; ENSSLIN; ENSSLIN, 2017). Dessa forma, na análise bibliométrica investigou-se: (1) autores com trajetória na área de Avaliação de Desempenho da Experiência do Usuário e suas áreas de atuação; (2) publicações mais relevantes sobre o tema; (3) periódicos científicos que têm devotado espaço à divulgação de pesquisas sobre o tema; e (4) palavras-chave que representam o tema na literatura científica.

Com esses dados observados, analisou-se (i) se os Sistemas de Avaliação e Gestão de Desempenho desenvolvidos/utilizados estão aptos a considerar o contexto e os objetivos para os quais a mensuração está realizada; e (ii) se tomam cuidado para não utilizar dados ou informações em um contexto diferente daquele em que foi concebido, pois isso tem implicações sobre os propósitos e usos da Avaliação de Desempenho, para identificar lacunas e propor futuras pesquisas. 


\section{Referencial teórico}

\subsection{Experiência do Usuário}

O consumo de experiências é um setor que tem apresentado crescimento significativo nos últimos anos, ocasionando um aumento no volume de estudos relacionado à área (PINE; GILMORE, 1999; RICHARDS, 2001; OH et al., 2007; FREIRE, 2009; DEMILIS, 2015). O Oxford English Dictionary define o termo experiência como "um evento ou ocorrência que deixa uma impressão em alguém". $\mathrm{Na}$ economia de experiência emergente, os consumidores procuram experiências únicas, além do simples consumo de produtos e serviços, porque o nível alto e consistente da qualidade dos produtos e serviços não pode mais ser usado como fator de diferenciação pelos usuários. Essa nova demanda por experiências únicas e memoráveis exige que as empresas desenvolvam uma provisão de valor agregado, distinta para produtos e serviços que já alcançaram um nível de qualidade funcional consistente e alto (PINE; GILMORE, 1999; OH et al. 2007). Portanto, definir e melhorar a experiência do usuário é uma crescente prioridade para pesquisas de mercado por esta estar substituindo a qualidade como aspecto competitivo para o marketing (KLAUS; MAKLAN, 2013).

A experiência se origina de um conjunto de interações complexas entre o usuário e uma empresa ou as ofertas de produtos da empresa (LASALLE; BRITTON 2003; HOLBROOK, 2006; CARÙ; COVA 2007). Os usuários também podem cultivar sua própria experiência única (PRAHALAD; RAMASWAMY 2004), na qual a empresa fornece artifícios e contextos para permitir ao consumidor moldar sua própria experiência (CARÙ; COVA 2003). Lemon e Verhoef (2016) conceituam UX como "jornada" de um cliente com uma empresa ao longo do tempo durante o ciclo de compra em vários pontos de contato, sendo um processo dinâmico. A UX é uma avaliação multidimensional e holística, formada por diferentes dimensões e fatores, mesmo que estes estejam apenas no subconsciente do usuário (SCHMITT, 1999; GENTILE; SPILLER; NOCI, 2007; HOSANY; WITHAM, 2009). Para Schmitt (1999), a experiência do cliente pode ser definida nestas dimensões: experiências sensoriais (sentido); experiências afetivas (sensação); experiências cognitivas criativas (pensamento); experiências físicas, comportamentos e estilos de vida (ato); e experiências de identidade social (relacionamento). Gentile, Spiller e Noci (2007) incluem um componente adicional, pragmático, definido como o ato prático de fazer algo (HOSANY; WITHAM, 2009).

A diversidade e a abrangência das experiências envolvem uma ampla gama de propriedades e métricas muito variadas, sendo, muitas vezes, particulares, efêmeras e subjetivas, contudo são também projetáveis (LAW et al. Al, 2009; HASSENZAHL, M; DIEFENBACH, S; GÖRITZ, 2010; BEST, 2012), porque são condicionadas por fatores humanos (GREEN e JORDAN, 1999). Isso impõe dificuldades à proposição dos conceitos e instrumentos que delimitem e identifiquem aspectos componentes da ampla e diversificada experiência do usuário. Isso também poderia justificar a diversidade de termos encontrados no presente estudo para Experiência do Usuário (em inglês, User Experience), como Customer Experience ou Brand Experience, usados fundamentalmente para se referir a mesma coisa, porém variando de acordo com a área em que é abordada.

Segundo Lemon e Verhoef (2016), o ressurgimento da experiência do cliente (usuário) e o foco nas jornadas de decisão do consumidor sugerem que as empresas estão ampliando seu pensamento sobre marketing e considerando como projetar e gerenciar todo o processo pelo qual o cliente passa. A satisfação do usuário pode ser um dos componentes da sua experiência, com foco na avaliação cognitiva do usuário da experiência (LEMON; VERHOEF, 2016). Na área do design, no início o interesse de seus profissionais foi voltado à experiência do usuário. A prioridade era 
antecipar idealmente as necessidades e soluções para promover experiências prático-funcionais positivas. Atualmente, o interesse é, primeiro, conhecer a realidade e a potencialidade experiencial do usuário, especialmente para investir na inovação ou no aprimoramento simbólico-afetivo das experiências. É por isso que se afirma o crescimento dos projetos de design orientados à experiência na atualidade OLIVEIRA, R. N.; LIMEIRA, C. D.; SANTA-ROSA, J. G, 2014; TONETTO; DESMET, 2016).

Assim, dado o valor da Experiência do Usuário e sua relação com o desempenho de uma organização, percebe-se a importância do estudo da Avaliação de Desempenho da Experiência do Usuário para uma empresa, marca, produto e/ou serviço.

\subsection{Avaliação de Desempenho}

A Avaliação de Desempenho (AD) é uma atividade complexa e consiste em um processo de quantificação da eficiência e eficácia das ações, por um conjunto de indicadores individuais, que formam um Sistema de Avaliação de Desempenho (NEELY; GREGORY; PLATTS, 1995). Ela tem por objetivo fornecer aos seus usuários informações relevantes e tempestivas, servindo de apoio à gestão e criando um potencial de aumentar a perspectiva de alcance aos resultados desejados, que podem estar relacionados, por exemplo, com fornecer ao usuário uma experiência significativa e memorável. Por isso, deve ser uma tarefa contínua que aborde questões relacionadas ao processo de negócio em um contexto organizacional (HALACHMI, 2005). Assim, os indicadores necessitam refletir o contexto estratégico intrínseco à organização, definidos internamente e agrupados em um conjunto (LEBAS, 1995; NEELY; GREGORY; PLATTS, 1995).

O processo de definição/construção dos indicadores tem caráter dinâmico, no qual a empresa aponta os indicadores que devem ser avaliados e de que forma serão mensurados, de modo que possam fornecer informações claras aos gerentes, supervisores e operadores, a fim de subsidiar as tomadas de decisão (GHALAYINI; NOBLE, 1996). Para isso, além do alinhamento à estratégia, é fundamental que haja entendimento de que o ambiente em que a organização está inserida se transforma e, consequentemente, a estratégia se altera, indicando que a forma de se avaliar o desempenho precisa acompanhar as transformações dos ambientes interno e externo (MELNYK et al., 2014; CARNEIRO-DA-CUNHA; HOURNEAUX; CORRÊA, 2016), estando elas alinhadas com as percepções, expectativas e necessidades do usuário. Além desses elementos, os modelos de AD construídos devem levar em consideração as percepções do decisor em um contexto específico (LEBAS, 1995), e, segundo defende a abordagem construtivista, devem promover a ampliação do entendimento sobre seu contexto de atuação (DUTRA et al., 2015).

\subsection{Avaliação de Desempenho da Experiência do Usuário}

Experiências são subjetivas, particulares, não podendo nunca serem iguais (HASSENZAHL, M; DIEFENBACH, S; GÖRITZ, 2010). Por isso, a dificuldade de se estabelecer uma métrica para mensurálas, avaliá-las e servir de instrumento de comunicação para gestão, contudo se pode categorizá-las (HASSENZAHL, M; DIEFENBACH, S; GÖRITZ, 2010). Nesse contexto, a Avaliação de Desempenho efetiva pode estimular a melhoria contínua e auxiliar na construção de indicadores de forma que sejam organizados em um Sistema de AD e forneçam feedback tempestivo aos gestores (LEBAS, 1995; NEELY; GREGORY; PLATTS, 1995).

Um produto projetado, sem entender a necessidade do usuário, é susceptível a perder oportunidades para uma experiência verdadeiramente memorável e os benefícios resultantes para a empresa por trás do produto (UNGER; CHANDLER, 2009). A UX é uma determinante-chave do 
comportamento do consumidor e um importante objetivo estratégico para as organizações (KLAUS; MAKLAN, 2013). Portanto, as respostas dadas pelos Sistemas de AD somente são efetivas se as métricas individuais estiverem alinhadas aos objetivos e às estratégias da organização (GHALAYINI; NOBLE, 1996; FERREIRA; OTLEY, 2009). As percepções dos clientes sobre sua experiência geralmente estão associadas à questão de como o produto, ou serviço, é entregue em vez de explorar o que é entregue (GOLDSTEIN et al., 2002) e como a experiência do cliente se relaciona com importantes resultados de marketing (KLAUS; MAKLAN 2013).

A medição da experiência do cliente desempenha um papel crítico na divulgação de ideias ativas para a empresa. Empresas de alto nível tentam medir e avaliar a experiência geral dos clientes com a empresa por meio de inúmeras métricas, embora não tenham sido desenvolvidas grandes escalas de avaliação da experiência do cliente, não havendo abordagens de medição robustas para avaliar todos os aspectos da UX (LEMON; VERHOEF, 2016). Portanto, a AD, sob uma perspectiva construtivista, pode auxiliar na construção de indicadores da UX e promover o acompanhamento e monitoramento deles, trazendo relevantes vantagens nesse contexto.

\section{Resultados}

\subsection{Análise Bibliométrica}

A primeira característica analisada diz respeito aos pesquisadores desse fragmento da literatura. Identificou-se que o Portfólio Bibliográfico consta de 194 autores. Os autores com maior ocorrência possuem de quatro a cinco artigos, e os demais somente dois artigos no PB.

Ao se identificar a variável Autores Prolíficos, os destaques do PB foram compostos por Maklan, Edvardsson e Klaus. Segundo os sites das universidades dos autores estudados, Maklan é professor titular em Marketing Estratégico, na Universidade de Cranfield, Escola de Administração. Ele pesquisa sobre Big Data - Marketing Analytics, CRM, Customer Experience, Marketing Measurement e Accountability e Marketing Leadership. Possui como parceiros, nas pesquisas desse PB, Uta Juttner, Philipp Klaus, Dorothea Schaffner, Katharina Windler. Já Edvardsson é professor de Administração de Empresas e Diretor do Centro de Pesquisa de Serviços, na Universidade de Karlstad, na Suécia. Suas principais áreas são a qualidade do serviço, desenvolvimento de serviços, infusão de serviços na fabricação, experiência em serviços e dinâmica de relacionamento. Possui como parceiros, nas pesquisas desse PB, Bo Enquist, Robert Johnston, Sara Sandström, Per Kristensson, Peter Magnusson, Ute Walter, Öström e Åsa. E Klaus é professor de Experiência de Cliente e Estratégia de Marketing na ESCEM School of Business and Management, França, visitante na Faculdade de Administração, Universidade de Cranfield, e é professor titular da Universidade LUMSA, em Roma (Itália) e Universidade de Valência (Espanha). Suas áreas de experiência incluem estratégia e gerenciamento de experiência do cliente, qualidade da experiência do cliente, estratégia de marketing, influência das atividades de marketing e experiência do cliente sobre o comportamento do consumidor. Possui como parceiro, nas pesquisas desse PB, Maklan.

Ressalta-se que Maklan e Klaus possuem áreas de pesquisa alinhadas ao tema, enquanto Edvardsson segue publicando na área da Experiência do Usuário, mas não está necessariamente ligado à área de serviços. Em continuidade, buscaram-se os autores que seriam destaques nas referências dos artigos do PB, respeitando as mesmas delimitações impostas na seleção do Portfólio. Na análise das referências, foi possível constatar que os autores prolíficos identificados no PB foram citados por outros autores do PB. Isso denota que os artigos desses autores se mostram importantes para a Avaliação de Desempenho da Experiência do Usuário. Ainda, voltaram a se 
destacar Maklan e Klaus. Contudo, observa-se também os autores mais citados nos artigos do Portfólio Bibliográfico e das referências. Katherine N. Lemon e Peter C. Verhoef são os mais citados, com 30 citações cada, porém apresentam apenas dois artigos tanto no PB, quanto nos artigos das referências. Os dois autores trabalharam em parceria produzindo os artigos do PB e das referências. Lemon é professora na Carroll School of Management, da Boston College. Suas pesquisas e ensino centram-se na experiência do cliente, na gestão de clientes, na equidade dos clientes, na estratégia de marketing e na dinâmica dos relacionamentos entre clientes e empresas. Já Verhoef é professor de Marketing, no Departamento de Marketing, da Faculdade de Economia e Negócios, da Universidade de Groningen, Países Baixos. Ele é diretor da Universidade de Groningen Business School. Os seus interesses de pesquisa dizem respeito à gestão de clientes e fidelização de clientes.

Dentre os autores de destaque do PB, predomina a área de atuação voltada para a Administração. Todos esses autores já têm uma trajetória de pesquisa sobre a experiência do cliente e o comportamento do consumidor. Também analisando os autores mais citados, foram observadas as áreas de Gestão e Marketing como suas áreas de formação e atuação. Nota-se que os autores que tiveram de dois a cinco artigos no PB possuem as seguintes áreas de atuação (Figura 3):

\section{ÁREAS DE ATUAÇÃODOSOSTTORES DE DESTAQUE}

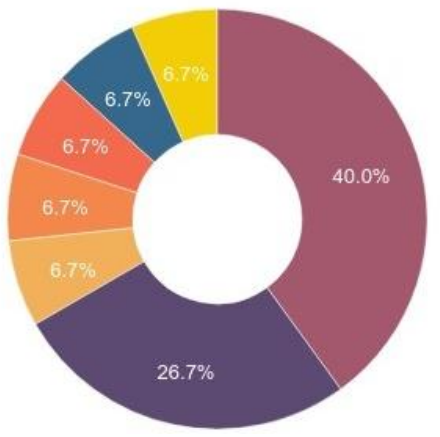

- Administração

- Marketing

- Psicologia

- Design-User Experience/Experience Design

- Turismo, Esporte e Hotelaria

- Gestão da hospitalidade

- Restaurante e Artes culinárias

Fonte: Elaborado pelos autores.

A segunda característica refere-se às publicações mais relevantes sobre o tema (Gráfico 1).

Gráfico 1. Relevância de artigos

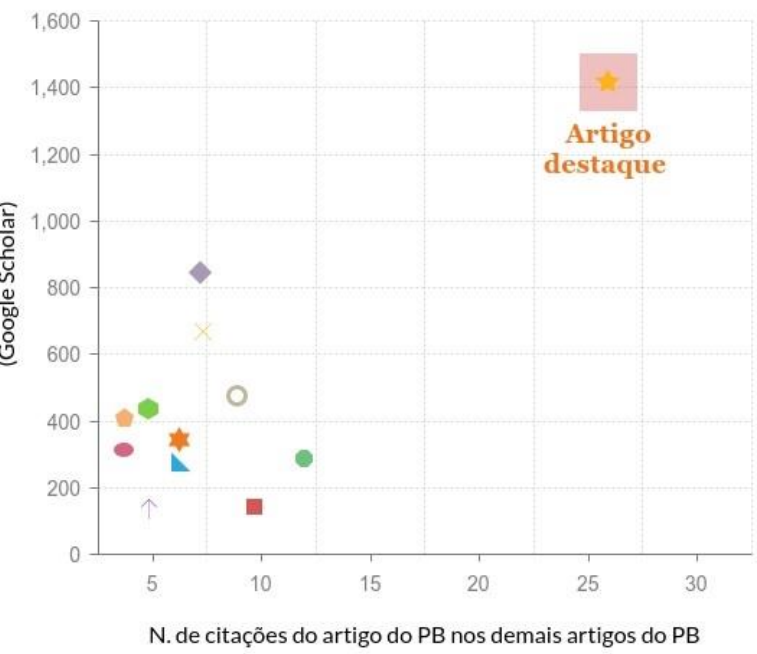

Verhoef, P.C. et al.

Klaus, P. and Maklan, S.

- Maklan, S. and Klaus, P.

O Hosany, S. and Witham, M.

Oh, H. et al.

Zomerdijk, L.G. and Voss, C.A.

- Iglesias, 0 . et al.

Lemon, K. N. and Verhoef, P.C.

Palmer, A.

Klaus, P. and Maklan, S.

Edvardsson, B. et al.

- Bigné, J. E. et al.

Fonte: Elaborado pelos autores. 
0 artigo Customer Experience Creation: Determinants, Dynamics and Management Strategies (2009) foi o mais citado no PB e nas referências, com 26 citações. É também o artigo com mais citações na literatura, 1.410 até o final de 2017. Além de ter como autores Verhoef e Lemon, também possui Parasuraman, Roggeveen, Tsiros, e Schlesinger como coautores, todos eles são doutores que pesquisam sobre experiência, atendimento e satisfação do cliente.

Pode-se perceber o baixo volume de artigos recentes publicados em periódicos, entre os autores mais citados no PB e nas referências, a respeito da AD da Experiência do Usuário. $O$ artigo mais recente, Understanding Customer Experience Throughout the Customer Journey, de Lemon e Verhoef, é de 2016 e está presente no PB. Outro artigo recente que analisa as publicações dos autores data de 2017, cujo autor é Stan Maklan, contudo ele não se enquadrou nas características observadas no processo de seleção do Portfólio Bibliográfico. As publicações identificadas dos outros autores estavam entre os anos de 2009 e 2013, também presentes no PB deste estudo.

Outra variável investigada foi a receptividade dos periódicos em relação ao tema pesquisado. O periódico que publicou mais artigos do PB foi o Journal of Travel Research (JTR), com seis artigos publicados. Os periódicos International Journal of Contemporary Hospitality Management (IJCHM), Journal of Service Management (JSMan) e Journal of Services Marketing (JSM) ficaram empatados em segundo lugar, com quatro artigos cada. Observa-se que o periódico americano JTR possui o maior fator de impacto (JCR 4.564) dentre esses quatro. Já o IJCHM possui fator de impacto JCR 3.196, o JSMan possui fator de impacto JCR 2.897 e o JSM possui fator de impacto JCR 1.811. Dentre os periódicos dos artigos do PB que mais se destacaram na literatura pelo número de citações, apenas o Journal of Travel Research (JTR) aparece com algum artigo entre os mais citados. Entre os artigos mais relevantes pelo número de citações no PB e nas referências, dos periódicos apontados como mais receptivos para a temática desta pesquisa, aparecem os journals JTR, JSMan e JSM.

Os quatro periódicos de destaque, citados anteriormente, são da área de Negócios, Gestão e Contabilidade, contudo o JTR possui como categoria a área de Turismo e Viagem; o IJCHM e o JSMan, Gestão de Turismo, Lazer e Hospitalidade; e o JSM, a categoria Marketing. Apesar de os autores de destaque serem predominantemente das áreas de Administração e Marketing, somente um dos periódicos que se destacou se classifica na categoria Marketing.

Nenhum dos artigos dos autores de destaque mencionados são voltados especificamente para categorias de Turismo, Viagens, Hospitalidade e afins. Muitos periódicos onde foram publicados artigos do PB também são voltados para áreas de Serviços, Marketing, Gestão. Contudo, é perceptível a carência de periódicos voltados para Projetos, Gestão de Projetos, Engenharia ou Design, o que poderia preencher uma carência de publicações voltadas para Avaliação de Desempenho da Experiência do Usuário publicados em periódicos sobre Projetos, Gestão de Projetos, Engenharia ou Design.

Já quando se observa os periódicos dos artigos das referências, pode-se notar uma variação nas categorias, surgindo mais periódicos voltados para Marketing, Operações, Logística e Qualidade, Gestão Estratégica, e Economia. Destacam-se os periódicos: Journal of Travel Research, Managing Service Quality: An International Journal, International Journal of Market Research, Journal of Brand Management, Journal of Hospitality \& Tourism Research, Journal of Marketing, Journal of Service Research e Journal of Services Marketing.

A Figura 4 apresenta uma síntese dos resultados das três variáveis investigadas. 
Figura 4. Síntese dos autores, artigos e periódicos de destaque.
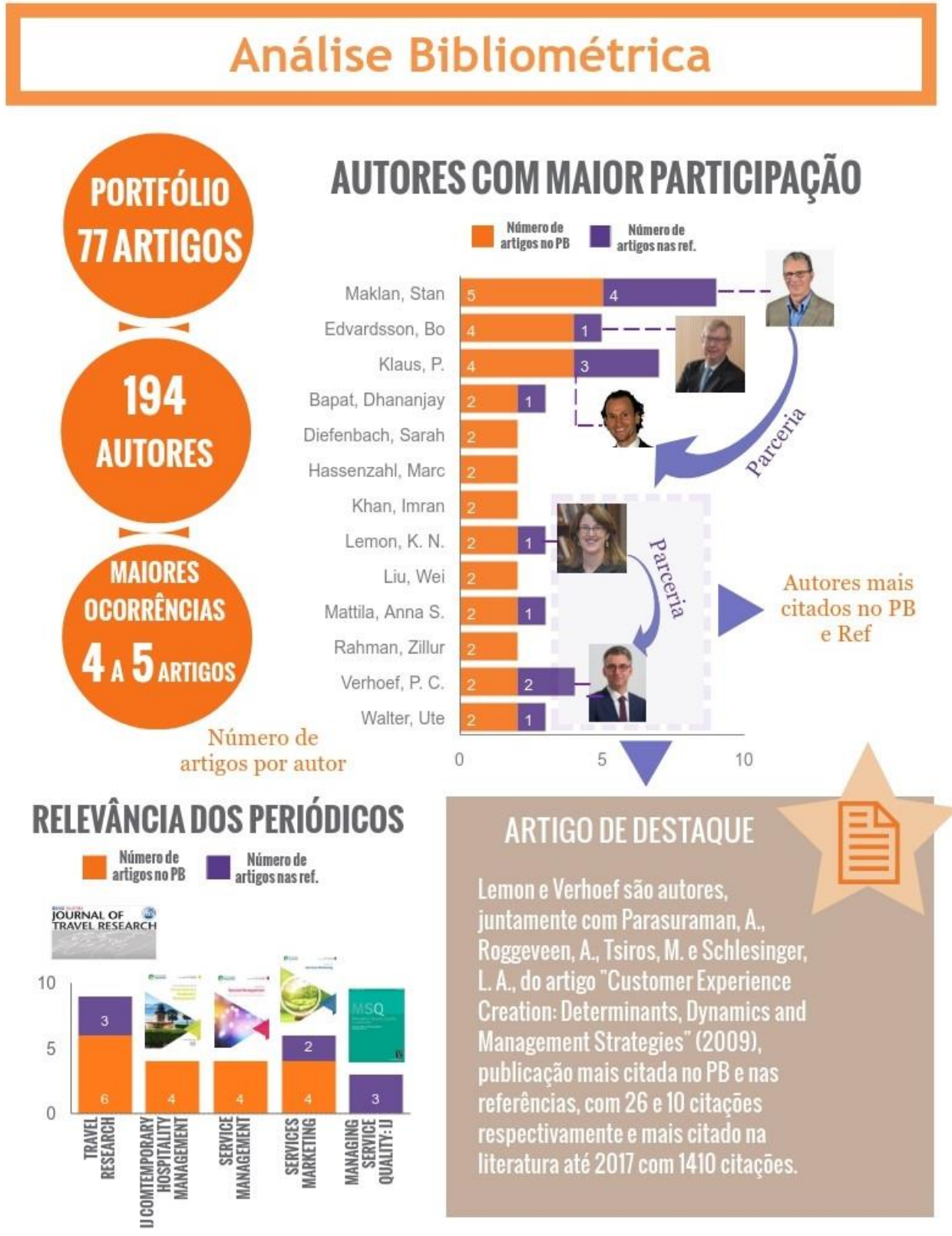

Fonte: Elaborado pelos autores.

Um fator também analisado foram as palavras-chave mais utilizadas pelos artigos e pelas referências. As palavras-chave mais observadas, tendo entre três e cinco incidências foram: customer experience, brand experience, customer experience management, emotions (Psychology) e Marketing. Esse resultado valida a aderência das palavras-chave definidas no início do processo de seleção dos artigos, apesar de apenas customer experience ser um dos termos usados na fase inicial desta pesquisa. Contudo, os outros termos são alinhados à temática, porém não necessariamente definem a Experiência do Usuário, portanto não sendo palavras que devessem ser usadas para definir o termo de busca inicial. A Figura 5 apresenta a rede de palavras-chave encontradas.

Com base no mapa de palavras-chave, destacaram-se alguns clusters pela incidência dos termos, força nas conexões, conexões e não conexões com outros termos. O fato de as UX serem 
subjetivas e particulares a cada indivíduo torna comum relacioná-las com aspectos subjetivos como emoções (emotion/emotions), percepções (perception), satisfação (satisfaction), estética (aesthetics) e comportamento do usuário (consumer behavior), e a presença de clusters com esses termos acaba por reforçar a relação entre UX e esses aspectos. Também se destacam clusters relacionados à fidelidade do usuário (brand loyalty e customer loyalty), reforçando a relação entre a experiência do usuário e a lealdade do cliente à marca ou à organização. O cluster desenvolvimento de escala (scale development) é outro que se destaca, o que poderia ser justificado por estudos que buscam escalas para avaliar o desempenho da UX.

Figura 5. Mapa de palavras-chave

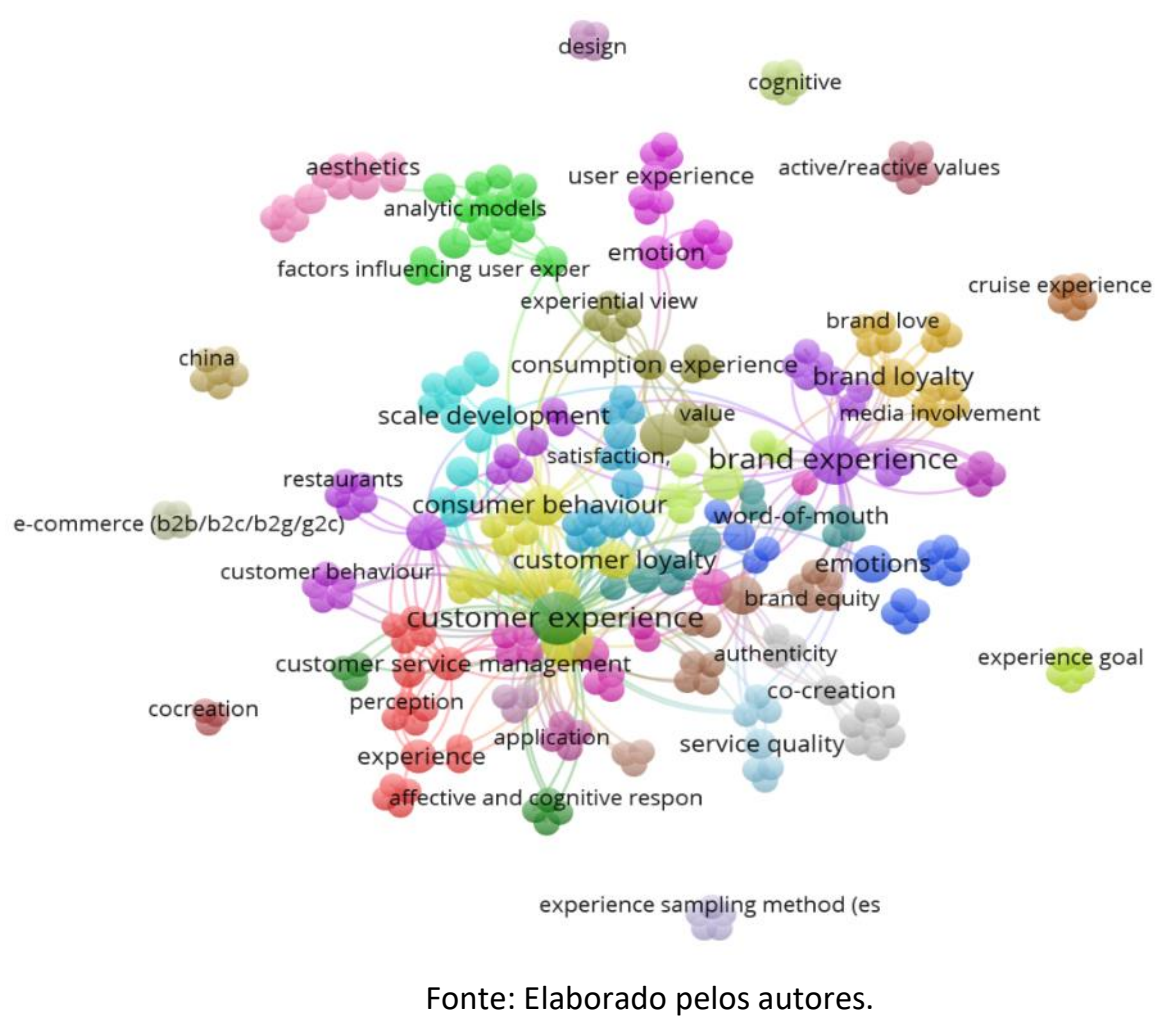

\subsection{Contribuições da pesquisa para o conhecimento científico: futuras investigações}

Com a análise dos resultados encontrados, pode-se perceber lacunas que podem promover avanços nessa área de conhecimento:

- Oportunidade de pesquisas na área de design que se voltem para a Avaliação de Desempenho/mensuração da experiência do usuário.

- Definição de um termo padrão e conceituação de Experiência do Usuário, haja vista a adoção de diversas expressões como user experience, customer experience, consumption experience, brand experience e experience.

- Observação e análise de aspectos da Experiência do Usuário e construção de indicadores alinhados à UX, que evidenciem e avaliem o que está sendo mensurado.

- Necessidade de Sistemas de Avaliação de Desempenho da experiência de caráter construtivista que possa se adaptar à realidade do desenvolvimento de projetos.

Com base na análise feita, é possível afirmar que as pesquisas na área de Avaliação de Desempenho da Experiência do Usuário carecem de reconhecer que 1) um termo padrão para UX, 
bem como aspectos que a compõem; 2) as informações mensuradas por Sistemas de Avaliação de Desempenho da UX são limitadas; e 3) deve-se promover estudos relacionados à UX nos processos de design, tendo em vista o valor e a importância dados a ela pelos usuários.

Por fim, observa-se que a Avaliação de Desempenho da Experiência do Usuário pode oferecer benefícios aos processos de desenvolvimento de produtos e serviços auxiliando na construção de indicadores, o que poderia influir no desempenho desse produto ou serviço no mercado e, assim, consequentemente, influir no desempenho de uma marca ou organização.

\section{Conclusões}

O presente estudo teve como objetivo investigar as pesquisas científicas, em língua inglesa, sobre Avaliação de Desempenho da Experiência do Usuário, a fim de identificar lacunas e oportunidades de pesquisa. Por meio da operacionalização do instrumento ProKnow-C, foram selecionados 77 artigos que formaram o PB. A análise bibliométrica revelou o periódico americano Journal of Travel Research como o mais receptivo ao tema, tendo o maior fator de impacto entre os periódicos de destaque. É voltado para a área de Negócios, Gestão e Contabilidade e se enquadra na categoria Turismo e Viagem. Turismo, Viagem, Lazer e Hospitalidade são uma forma de prestação de serviço que fornece ao usuário um benefício predominantemente intangível, uma experiência (MAGER, 2009), o que poderia justificar a incidência de artigos relacionados à experiência do usuário em periódicos dessas áreas.

Dos 194 autores dos artigos do PB, destacam-se três autores prolíficos: Stan Maklan, Edvardsson Bo e Philipp Klaus. Contudo, analisando seus históricos e linhas de pesquisa, não se pode afirmar que possuem trajetória extensa e totalmente alinhada à área de Avaliação de Desempenho da Experiência do Usuário. Maklan e Klaus também se destacaram como autores dos artigos das referências. Outros autores que também se destacaram foram Katherine Lemon e Peter Verhoef como os mais citados, com 30 citações no PB cada, mesmo apresentando apenas dois artigos tanto no PB, quanto nas referências. Dessas citações, 26 delas, o que corresponde a $34 \%$ do PB, se referiam ao artigo Customer Experience Creation: Determinants, Dynamics and Management Strategies, de 2009. Esse artigo, também escrito por Parasuraman, Roggeveen, Tsiros e Schlesinger, é a publicação do PB que possui o maior número de citações na literatura (Google Scholar). Isso demostra que o artigo tem grande relevância para a temática, sendo considerado um artigo de referência quando se trata sobre Avaliação de Desempenho da Experiência do Usuário.

Com relação às palavras-chave dos artigos do PB e o mapa gerado, pode-se perceber a maleabilidade do termo user experience, surgindo diversas variações para o mesmo conceito, tais como: customer experience, brand experience, consumption experience, bem como as derivações consumer experience quality, user experience frameworks, user experience models, cruise experience e event experience. Com base no mapa das palavras-chave, destacaram-se de alguns clusters pela incidência dos termos, força nas conexões, conexões e não conexões com outros termos. Salienta-se o cluster design por não apresentar conexões com os principais clusters ao centro do mapa. Como apontado anteriormente, não se destacaram autores voltados para as áreas de atuação do design, mas, sim, para as áreas de gestão organizacional. Assim, pode-se perceber a carência de pesquisas que tenham como eixo central estudos relacionando design, desenvolvimento de projeto e engenharia à UX.

Com base na leitura do PB, identificou-se as seguintes lacunas: observação e análise de aspectos da UX e construção de indicadores; modelos propostos e dados mensurados por Sistemas 
de Avaliação de Desempenho da UX são limitadas; carência de Sistemas de Avaliação de Desempenho da experiência de caráter construtivista que possa se adaptar à realidade do desenvolvimento de projetos; e falta de pesquisas na área de design e afins voltadas para a Avaliação de Desempenho/mensuração da UX. Sugerem-se, para trabalhos futuros: a ampliação deste estudo a outras bases de dados; e o desenvolvimento de trabalhos que visem eliminar as lacunas apontadas na seção 4.2 .

\section{Referências}

BEST, K. Fundamentos de Gestão do Design. Bookman Editora, 2012.

BROWN, M.; HYATT, D.; BENSON, J. Consequences of the performance appraisal experience. Personnel Review, v. 39, n. 3, p. 375-396, 2010.

CARNEIRO-DA-CUNHA, J. A.; HOURNEAUX JR, F.; CORRÊA, H. L. Evolution and chronology of the organizational performance measurement field. International Journal of Business Performance Management, v. 17, n. 2, p. 223-240, 2016.

CARÙ, A.; B. COVA. Consuming Experience. London: Routledge. 2007.

CASTRO, C. M. A prática da pesquisa. McGraw-Hill, 1977.

CRESWELL, J. W. Research design: qualitative, quantitative, and mixed methods approaches. Sage Publications, 2014.

DEMILIS, M. P. Fatores Humanos no Design de serviços: Valoração de aspectos da experiência de consumo pelo público idoso em supermercados. Florianópolis, 2015. 135 p. Dissertação (Mestrado em Design) - Programa de Pós-Graduação em Design - PPGDesign, Universidade do Estado de Santa Catarina, 2015.

DUTRA, A.; RIPOOL-FELIU, V. M. R.; FILLOL, A. G.; ENSSLIN, S. R.; ENSSLIN, L. The construction of knowledge from the scientific literature about the theme seaport performance evaluation. International Journal of Productivity and Performance Management. v. 64, iss 2, p. 243-269, 2015.

ENSSLIN, L.; ENSSLIN, S. R.; PINTO, H. M. Processo de investigação e análise bibliométrica: avaliação da qualidade dos serviços bancários. Revista de Administração Contemporânea, v. 17, n. 3, p. 325349, 2013.

ENSLLIN, S. R.; ENSSLIN, L.; IMLAU, J. M.; CHAVES, L. C. Processo de mapeamento das publicações científicas de um tema: portfólio bibliográfico e análise bibliométrica sobre Avaliação de Desempenho de cooperativas de produção agropecuária. Revista de Economia e Sociologia Rural (Impresso), v. 52, n. 03, p. 587-608, 2014.

FERREIRA, A.; OTLEY, D. The design and use of performance management systems: An extended framework for analysis. Management Accounting Research, v. 20, n. 4, p. 263-282, 2009

FREIRE, K. M. Reflexões sobre o conceito de design de experiências. SDRJ, v. 2, p. 37-44, 2009.

GENTILE, C.; SPILLER, N.; NOCl, C. How to sustain the customer experience: an overview of experience components that co-create value with the customer. European Management Journal, v. 25 n. 5, p. 395-410. 2007.

GHALAYNI, A. M.; NOBLE, J. S. The changing basis of performance measurement. International Journal of Operations \& Production Management, v. 16, n. 8, p. 63-80, 1996. 
GOLDSTEIN, S.M.; JOHNSTON, R.; DUFFY, J.; RAO, J. The service concept: the missing link in service design research? Journal of Operations Management, 20, 2, p. 121-134. 2002.

GREEN, W.; JORDAN, P. W. Human factors in product design: current practice and future trends. CRC Press, 1999.

HALACHMI, A. Performance measurement is only one way of managing performance. International Journal of Productivity and Performance Management, v. 54, n. 7, p. 502516, 2005.

HASSENZAHL, M.; SCHÖBEL, M.; TRAUTMANN, T. How motivational orientation influences the evaluation and choice of hedonic and pragmatic interactive products: The role of regulatory focus. Interacting with Computers, v. 20, n. 4/5, p. 473-479, 2008.

HASSENZAHL, M; DIEFENBACH, S; GÖRITZ, A. Needs, affect, and interactive products-Facets of user experience. Interacting with computers, v. 22, n. 5, p. 353-362, 2010.

HOLBROOK, M. Book reviews: the consumption experience. J. of Macromarketing, v. 26 n. 2, p. 25966. 2006.

HOSANY, S.; WITHAM, M. Dimensions of cruisers' experiences, satisfaction, and intention to recommend. Journal of Travel Research, v. 49, n. 3, p. 351-364, 2010.

KLAUS, P.; MAKLAN, S. Towards a better measure of customer experience. 2013.

LACERDA, R. T.O.; ENSSLIN, L.; ENSSLIN, S. R. Uma análise bibliométrica da literatura sobre estratégia e avaliação de desempenho. Gestão \& Produção, v. 19, n. 1, p. 59-78, 2012.

LACERDA, R.; ENSSLIN, L.; ENSSLIN, S. R. Research opportunities in strategic management field: a performance measurement approach. I. J. of Business Performance Management, v. 15, n. 2, p. 158174,2014

LASALLE, D.; BRITTON, T. Priceless: Turning Ordinary Products into Extraordinary Experiences. Harvard Business School Press, Boston, MA. 2003.

LAURANS, G.F.G.; DESMET, P.M.A.; HEKKERT, P. Assessing emotion in interaction: some problems and a new approach. In: Guenand, A. (Ed.), Proceedings of the 4th International Conference on Designing Pleasurable Products and Interfaces, Compiegne, France. October 13-16, 2009.

LAW, E.; ROTO, V.; HASSENZAHL, M.; VERMEEREN, A.; KORT, J. Understanding, scoping and defining user experience: a survey approach. In: CHI 2009. ACM, Boston, USA, p. 719-728, April 2009.

LEBAS, M. J. Performance measurement and performance management. International Journal of Production Economics, v. 41, n. 1-3, p. 23-35, 1995.

LEMON, K. N.; VERHOEF, P. C. Understanding customer experience throughout the customer journey. Journal of Marketing, v. 80, n. 6, p. 69-96, 2016.

MELNYK, S. A.; BITITCI, U.; PLATTS, K.; TOBIAS, J.; ANDERSEN, B. Is performance measurement and management fit for the future?. Management Accounting Research, v.25, n.2, p.173-186, 2014.

NEELY, A.; GREGORY, M. J.; PLATTS, K. Performance measurement system design: A literature review and research agenda. International Journal of Operations \& Production Management, v. 15, n. 4, p. 80-116, 1995.

$\mathrm{OH}, \mathrm{H}$.; FIORE, A. M.; JEOUNG, M. Measuring experience economy concepts: Tourism applications. 
Journal of Travel Research, v. 46, n. 2, p. 119-132, 2007.

OLIVEIRA, R. N.; LIMEIRA, C. D.; SANTA-ROSA, J. G. A experiência do usuário no processo evolutivo do design. Blucher Design Proceedings, v. 1, n. 4, p. 3451-3460, 2014.

PINE, J.; GILMORE, J. H. The experience economy: Work is theatre and every business a stage. Boston: Harvard Business School Press. 1999.

POELS, K.; DEWITTE, S. How to capture the heart? Reviewing 20 years of emotion measurement in advertising. J. Advert. Res. v. 46, n. 1, p. 18-37, 2006.

PRAHALAD, C. K.; V. RAMASWAMY. Co-Creation Experiences: The Next Practice in Value Creation. Journal of Interactive Marketing, v. 18, n. 3, p. 5-14, 2004.

RICHARDS, G. The Experience Industry and the Creation of Attractions. In Cultural Attractions and European Tourism, edited by G. Richards. Oxfordshire, UK: CABI Publishing, p. 55-69, 2001.

RICHARDSON, R. J. Pesquisa Social: métodos e técnicas. 3. ed. São Paulo: Atlas, 1999.

SCHMITT, B. H. Experiential Marketing. New York: The Free Press, 1999.

THIEL, G.; ENSSLIN, S.R.; ENSSLIN, L. Street Lighting Management and Performance Evaluation: Opportunities and Challenges. Lex Localis - Journal of Local Self-Government, v. 15, n. 2, p. 303-328, 2017.

TONETTO, L. M.; DESMET, P. M. A. Why we love or hate our cars: A qualitative approach to the development of a quantitative user experience survey. Applied Ergonomics, v. 56, p. 68-74, 2016.

UNGER, R. CHANDLER, C. A Project guide to UX design: for user experience designers in the fild or in the making. 1.ed. Berkeley, CA: New Riders, 2009.

VALMORBIDA, S. M. I.; ENSSLIN, S. R. Performance Evaluation of University Rankings: Literature Review and guidelines for future research. I. J. of Business Innovation Research. v. 14, n. 4, p. 479501, 2017.

\section{Agradecimentos}

Agradecemos à Coordenação de Aperfeiçoamento de Pessoal de Nível Superior (CAPES), ao Conselho Nacional de Desenvolvimento Científico e Tecnológico (CNPq), à Fundação de Amparo à Pesquisa e Extensão Universitária (FAPEU), à Pró-Reitoria de Extensão e Assuntos Comunitários (Proex), à Rede de Pesquisa e Desenvolvimento de Tecnologia Assistiva (RPDTA) e ao Programa de Pós-Graduação em Design (POSDESIGN/UFSC). 